\title{
Migration of a Hem-o-Lok Clip to the Ureter Following Laparoscopic Partial Nephrectomy Presenting With Lower Urinary Tract Symptoms
}

\author{
Kyung Seo Park, Young Jin Sim ${ }^{1}$, Han Jung ${ }^{1}$ \\ Department of Urology, Hyun-Dai UVIS Hospital, Incheon; \\ ${ }^{1}$ Department of Urology, Gachon University Gil Medical Center, Gachon University of Medicine and Science, Incheon, Korea
}

\begin{abstract}
We report a case of ureteral migration of a surgical clip after partial nephrectomy in which the clip was misdiagnosed as a ureteral stone. A 37-year-old woman had undergone laparoscopic partial nephrectomy of right renal cell carcinoma at another hospital 2 years previously. Postoperatively, she had gradually acquired lower urinary tract symptoms. Then, she complained of sudden right flank pain for a week. A plain X-ray and enhanced abdominopelvic computed tomography scan were performed. A $0.5 \mathrm{~cm} \times 1.0$ $\mathrm{cm}$ right upper ureteral opacity with borderline hydronephrosis was seen but could not be found on the X-ray. Ureteroscopy revealed a medium-sized Hem-o-Lok clip on the right upper ureter that was removed with a stone basket. We concluded that a Hem-o-Lok clip used for collecting system sealing had migrated to the ureter and had been misdiagnosed as a ureteral stone on a computed tomography scan.
\end{abstract}

Keywords: Nephrectomy; Surgical instruments; Ureteral calculi

Laparoscopic partial nephrectomy (LPN) has been performed for the treatment of small renal masses for nephron sparing in recent years and has results similar to those of open surgery in terms of outcome, such as positive surgical margin rates [1]. Although LPN results in enhanced perioperative patient safety compared with open partial nephrectomy (OPN) in the United States [2], it is a challenging and highly advanced laparoscopic procedure owing to the difficulty of securing the collecting system and suturing the renal defect. Furthermore, there is no gold standard single agent or combination of products that can be applied in all cases, but rather hemostatic agents such as glues, bolsters and argon laser are used either alone or in combination, as well as sutures [3].

In this report, we present a case of migration of a surgical clip (Hem-o-Lok clip; Weck, Teleflex Medical, Research Triangle Park, NC, USA) on the ureter after LPN in which the clip was initially misdiagnosed as a ureteral stone.

\section{CASE REPORT}

A 37-year-old woman presented to us complaining of sudden right flank pain and lower abdominal pain that had lasted for 1 week, as well as nausea and vomiting. Two years previously she had undergone a laparoscopic right partial nephrectomy at another hospital owing to a small right renal mass that was diagnosed as renal cell carcinoma (T1aN0M0). Postoperatively, she had gradually acquired lower urinary tract symptoms, such as urge incontinence, urgency, frequency, and nocturia. Three months earlier, she had been checked with abdominopelvic computed tomography (CT) for regular follow-up of the renal cell carcinoma and heard that there were no definite abnormal findings in the CT scan. In the physical examination, right costover-
Corresponding author: Han Jung

Department of Urology, Gachon University Gil Medical Center,

Gachon University of Medicine and Science, 21 Namdong-daero 774beon-gil,

Namdong-gu, Incheon 405-760, Korea

Tel: +82-32-460-3331, Fax: +82-32-460-8340, E-mail: urohana@gilhospital.com

Submitted: March 6, 2013 / Accepted after revision: June 19, 2013
This is an Open Access article distributed under the terms of the Creative Commons Attribution Non-Commercial License (http://creativecommons.org/licenses/by-nc/3.0/) which permits unrestricted non-commercial use, distribution, and reproduction in any medium, provided the original work is properly cited. 
tebral angle tenderness was observed. The results of a urine test showed 5 to 10 red blood cells per high power field, and plain $\mathrm{X}$-ray of the kidney, ureter, and bladder revealed metallic surgical clips on the right upper abdomen and a possible right renal stone, but no abnormal density on the ureteral courses (Fig. 1). The abdominopelvic CT scan revealed a $0.5 \mathrm{~cm} \times 1.0 \mathrm{~cm}$ opacity on the right proximal ureter with borderline hydronephrosis and a tiny right renal stone (Fig. 2). Ureteroscopy with the pa-

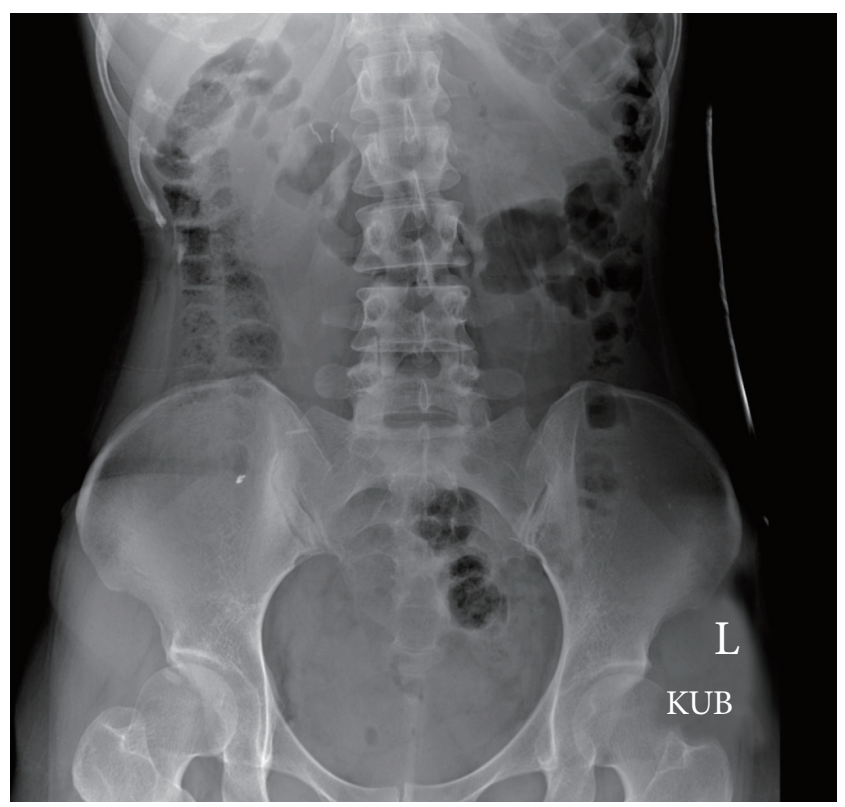

Fig. 1. Plain X-ray of the abdomen revealed metallic surgical clips on the right upper abdomen and a possible right renal stone but no abnormal density on the ureteral courses. KUB, kidney-ureter-bladder. tient under general anesthesia showed a white rectangular parallelepiped foreign body at the proximal right ureter (Fig. 3A). The foreign body was removed by use of a ureteroscopic stone basket device and was identified as a medium-sized surgical clip (Fig. 3B). There was no extravasation of the renal pelvis during contrast media instillation via the channel of the ureteroscope. A ureteral stent was placed for 1 week, and the patient had no more flank pain.

\section{DISCUSSION}

Currently, partial nephrectomy is considered a standard treatment for small renal tumors with the benefit of preserving renal function; improving overall survival, especially for patients younger than 65 years of age; and decreasing the overall mor-

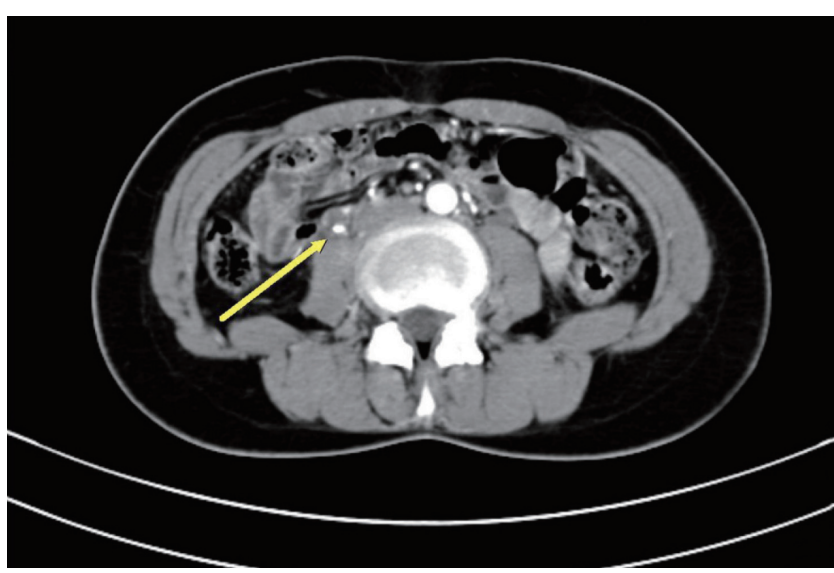

Fig. 2. abdominopelvic computed tomography scan revealed a $0.5 \mathrm{~cm} \times 1.0 \mathrm{~cm}$ opacity (arrow) on the right proximal ureter.
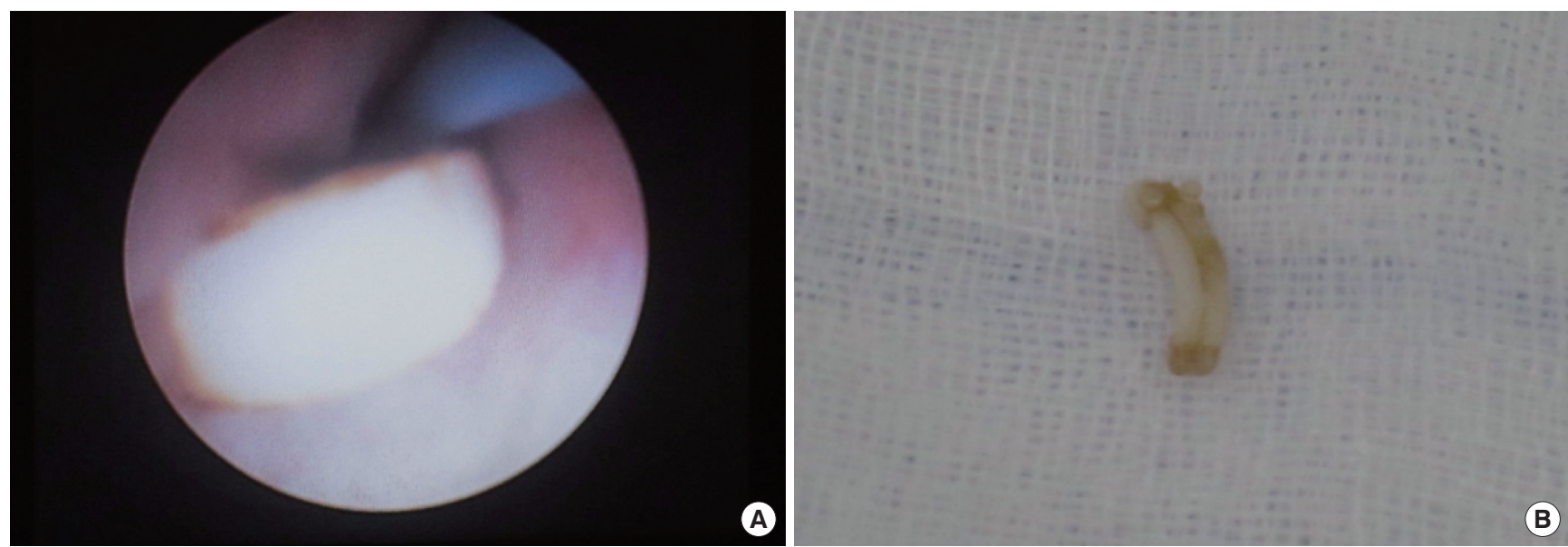

Fig. 3. (A) Ureteroscopy showed a white rectangular parallelepiped foreign body at the proximal right ureter. (B) The foreign body was removed by use of a ureteroscopic stone basket device and was identified as a medium-sized Hem-o-Lok Clip. 
tality rate. For T1b tumors, more clinical data are required to establish the oncological and functional benefits of partial nephrectomy (PN). LPN has come to represent comparable perioperative and oncological outcomes in the recent era [4] and has gained popularity, although it remains a challenging and highly advanced laparoscopic procedure. The most demanding step during LPN is the repairing of the collecting system and renal defect because this repair requires advanced laparoscopic skills and is performed under time pressure to minimize the warm ischemia time.

Ureteral migration of suture material after PN is not a common complication. There is a report of the migration of absorbable Lapra-Ty suture clips in the collecting system after LPN [5], and Massoud [6] also reported the migration of a metal surgical clip into the ureter after OPN, all of which were passed spontaneously. Furthermore, intravesical migration and stone formation of a surgical clip after laparoscopic radical prostatectomy has been reported [7], but there have been no reports of ureter migration of a surgical clip after partial nephrectomy.

Msezane et al. [3] reviewed the different sealants and laparoscopic instruments that are available for achieving hemostasis of the renal parenchyma in LPN and determined that there is no gold standard single agent or combination that can be applied to all cases. The decision as to which technology to use and how to manage the hilum should be made on a case by case basis.

Hem-o-Lok or metal clips that are used to repair the collecting system and the renal defect in LPN can migrate postoperatively and cause secondary complications, such as urinary stones. Ureteral stones following LPN can be managed conservatively with hydration and narcotics, but if symptoms do not improve, surgeons may consider more aggressive ureteroscopic management. Furthermore, the surgeon must be aware of the possibility of clip migration.

\section{CONFLICT OF INTEREST}

No potential conflict of interest relevant to this article was reported.

\section{REFERENCES}

1. Marszalek M, Meixl H, Polajnar M, Rauchenwald M, Jeschke K, Madersbacher S. Laparoscopic and open partial nephrectomy: a matched-pair comparison of 200 patients. Eur Urol 2009;55:1171-8.

2. Parsons JK, Palazzi K, Chang D, Stroup SP. Patient safety and the diffusion of surgical innovations: a national analysis of laparoscopic partial nephrectomy. Surg Endosc 2013;27:1674-80.

3. Msezane LP, Katz MH, Gofrit ON, Shalhav AL, Zorn KC. Hemostatic agents and instruments in laparoscopic renal surgery. J Endourol 2008;22:403-8.

4. Lee SY, Choi JD, Seo SI. Current status of partial nephrectomy for renal mass. Korean J Urol 2011;52:301-9.

5. Miller M, Anderson JK, Pearle MS, Cadeddu JA. Resorbable clip migration in the collecting system after laparoscopic partial nephrectomy. Urology 2006;67:845.e7-8.

6. Massoud W. Spontaneous migration of a surgical clip following partial nephrectomy. Urol J 2011;8:153-4.

7. Tugcu V, Polat H, Ozbay B, Eren GA, Tasci AI. Stone formation from intravesical Hem-o-lok clip migration after laparoscopic radical prostatectomy. J Endourol 2009;23:1111-3. 